\title{
Does the number of chromosomes matter?
}

\section{CRISPR-Cas9 enables successive fusions of 16 native chromosomes into one artificial single chromosome.}

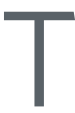
he number of chromosomes can range from one to hundreds for different eukaryotic species. Yet, it remains unknown how nature selects the number of chromosomes and whether there is biological 'meaning' behind the number.

Zhongjun Qin and his team from Shanghai Institute of Plant Physiology and Ecology were intrigued by these questions. They speculated that chromosome number might have minimal effects on cell growth. The team, together with collaborators including Guoping Zhao and Jin-Qiu Zhou, started a project five years ago to explore the possibility of artificially reducing the number of chromosomes in the eukaryotic model organism Saccharomyces cerevisiae, which has maintained its 16 chromosomes for millions of years.

Qin and his collaborators initially attempted to use traditional recombination techniques to edit the chromosomes, which was “very inefficient," comments Qin. The advent of the CRISPR-Cas9 system provided the opportunity to edit the yeast chromosomes in a practical manner.

In S. cerevisiae, each chromosome has two telomeres at both ends, as well as one centromere for normal chromosome segregation during cell division. To create a single chromosome from 16 chromosomes, they started by fusing 2 chromosomes and then repeated this fusion step for 15 rounds. They removed one telomere from each chromosome to promote end-to-end joining and simultaneously deleted one centromere to avoid dicentric chromosomes. This pairwise fusion can be iterated to elongate a chromosome and reduce the number of chromosomes.

During the pilot pairwise fusion experiments, Qin's team found that the order of the chromosome was not vital for yeast growth. "We actually random-paired the 16 chromosomes into eight groups. In the eight pairwise fusions, all the yeasts were healthy," says staff scientist Xiaoli Xue.

After the successful fusion experiments, they used the Hi-C method to study the 3D genome architecture of four yeast strains that possess varied numbers $(1,2,9$, and 16 , respectively) of chromosomes. Because of the gradual removal of centromeres and telomeres, the four yeast strains showed independent, distinct intrachromosomal interactions. The changes in chromosome number also remarkably altered the interchromosomal interactions: singlechromosome yeast lacked $99.7 \%$ of the interchromosomal interactions present in the parental strain. The overall genome structures in the 16- and 9-chromosome strains had a typical Rabl configuration, whereas the singleand double-chromosome strains showed a relatively twisted, globular configuration.

Beyond chromosomal 3D structure analysis, the teams also examined the transcriptome profile of the singlechromosome yeast. Surprisingly, they found that its transcriptome was nearly identical to that of the parental strain. Only 28 genes were differentially expressed in the singlechromosome yeast, of which 8 upregulated genes were associated with stress response. This upregulation indicates that a potential burden may be introduced by replication of the giant chromosome.

Despite the potential replication burden, the giant single chromosome had a minimal effect on cell growth, as Qin speculated. The size and shape, as well as the pattern of cell-cycle progress, of single-chromosome yeast resembled that of the parental strain, but with a slightly reduced growth rate.

However, the single-chromosome yeast did have functional defects. Cells showed weak competitiveness when cocultured with wild-type yeast. The researchers also observed reduced gamete production and viability in meiosis, although the singlechromosome haploid cells could mate and generate diploid cells to produce viable spores. Qin comments that "the artificial life still could not compete with the natural life; however, this is the V.1 of artificial yeast. The V.2 and the V.3 are on the way to come."

\section{Lei Tang}

Published online: 1 October 2018 https://doi.org/10.1038/s41592-018-0164-2

\section{SARSTEDT}

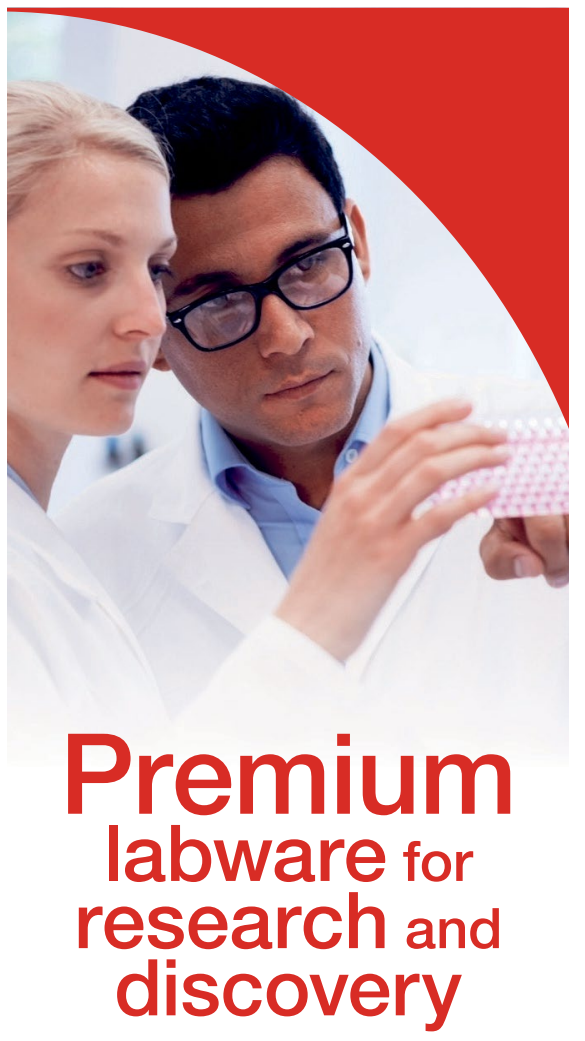

\section{Cell and Tissue Culture}

- Three different color coded growth surfaces

- Optimized, user-friendly geometries

- Labeling of all products with LOT number and expiration date

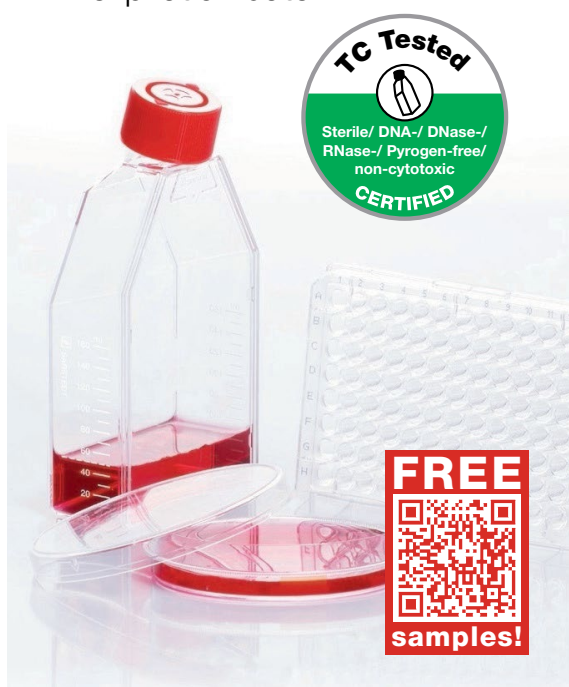

www.sarstedt.com

customerservice@sarstedt.us 